\title{
Topical Corticosteroid Therapy for Facial Acneiform Eruption due to EGFR Inhibitors in Metastatic Colorectal Cancer Patients: A Randomized Controlled Trial Comparing Starting with a Very Strong or a Weak Topical Corticosteroid (FAEISS Study, NCCH1512, Colorectal Part)
}

Katsuko Kikuchi ( $\sim$ kkikuchi@derma.med.tohoku.ac.jp )

Tohoku University School of Medicine: Tohoku Daigaku Daigakuin Igakukei Kenkyuka Igakubu Naoya Yamazaki

National Cancer Center Hospital: Kokuritsu Gan Kenkyu Center Chuo Byoin

Keiko Nozawa

Mejiro University: Mejiro Daigaku

Haruhiko Fukuda

National Cancer Center Hospital: Kokuritsu Gan Kenkyu Center Chuo Byoin

Taro Shibata

National Cancer Center Hospital: Kokuritsu Gan Kenkyu Center Chuo Byoin

Ryunosuke Machida

National Cancer Center Hospital: Kokuritsu Gan Kenkyu Center Chuo Byoin

Tetsuya Hamaguchi

Saitama Medical University International Medical Center: Saitama Ika Daigaku Kokusai Iryo Center

Atsuo Takashima

National Cancer Center Hospital: Kokuritsu Gan Kenkyu Center Chuo Byoin

Hirokazu Shoji

National Cancer Center Hospital: Kokuritsu Gan Kenkyu Center Chuo Byoin

Narikazu Boku

National Cancer Center Hospital: Kokuritsu Gan Kenkyu Center Chuo Byoin

Sumiko Takatsuka

Niigata cancer center hospital

Tatsuya Takenouchi

Nigata cancer center hospital

Tomohiro Nishina

National Hospital Organization Shikoku Cancer Center

Shusuke Yoshikawa 
Shizuoka Cancer Center Hospital

\section{Masanobu Takahashi}

Tohoku University Hospital: Tohoku Daigaku Byoin

\section{Akiko Hasegawa}

Osaka International Cancer Institute: Osaka Kokusai Gan Center

\section{Akihito Kawazoe}

National Cancer Center-Hospital East: Kokuritsu Gan Center Higashi Byoin

\section{Toshiki Masuishi}

Aichi cancer center hospital

\section{Hitoshi Mizutani}

Mie University Hospital: Mie Daigaku Igakubu Fuzoku Byoin

\section{Yoshio Kiyohara}

Shizuoka Cancer Center Hospital

\section{Research Article}

Keywords: epidermal growth factor receptor (EGFR), anti-EGFR antibody, colorectal cancer, skin toxicity, acneiform eruption, topical corticosteroid

Posted Date: August 30th, 2021

DOI: https://doi.org/10.21203/rs.3.rs-796362/v1

License: (c) (i) This work is licensed under a Creative Commons Attribution 4.0 International License. Read Full License

Version of Record: A version of this preprint was published at Supportive Care in Cancer on February 3rd, 2022. See the published version at https://doi.org/10.1007/s00520-022-06874-1. 


\section{Abstract}

Background: Althoughpre-emptive therapy with oral tetracycline, moisturizer, sunscreen and topical corticosteroid isuseful for preventing acneiform eruption (AfE) due to epidermal growth factor receptor (EGFR) inhibitors, no studies have examinedthe efficacy of topical corticosteroids themselves, or investigated the optimal strength of the corticosteroid for treating facial AfE (FAfE).

Patients and Methods: Screened patients with RAS wild-typecolorectal cancerstarted pre-emptive therapy with oral minocycline and moisturizeron initiation of cetuximab or panitumumab therapy. Patients who developed grade 1 or 2 FAfEwere randomly allocated to two groups:a ranking-down (RD) group,started with a very strong corticosteroid, and serially ranked down every 2 weeks unless FAfE exacerbated, and a ranking-up $(\mathrm{RU})$ group, started with a weak corticosteroid and serially ranked up at exacerbation. FAfE grade, patient quality of life, and adverse events (AEs) with topical corticosteroid were evaluated every 2 weeks. The primary endpoint was the total number of times grade 2 or higher FAfEidentified in thecentral review of the 8-weektreatment period.

Results: No significant differences in total numbers of grade 2 or higher FAfE and inAEs caused by topical corticosteroids were observedbetweengroups during the 8-week. Incidence of grade 2 or higher FAfEwas tended to lower inthe RD group during the first 2 weeks.

Conclusion: Considering long-term care of FAfE, the RU regimen appears suitableand should be considered the standard treatment for FAfEdue to EGFR inhibitor therapy.

Trial registration: UMIN Clinical Trials Registry (UMIN000024113)

\section{Introduction}

Epidermal growth factor receptor (EGFR) inhibitors including monoclonal antibodies (mAbs) for EGFR and tyrosine kinase inhibitors (TKIs) are effective therapeutic options for patients with colorectal cancer (CRC), non-small cell lung cancer (NSCLC) and squamous cell carcinoma of head and neck (SCCHN). Anti-EGFR mAbs such as cetuximab and panitumumab have been used for metastatic CRC as monotherapies or in combination with other anti-cancer agents. Patients receiving EGFR inhibitors commonly present with skin toxicities, including acneiform eruption (AfE), which sometimes cause discontinuation of the therapy. AfE develops as early as weeks 1-3 after initiating the therapy and reaches peak frequency by weeks 3-5 [1]. In particular, facial AfE (FAfE) can cause psychological distress and impair patient quality of life (QOL). In the STEPP [2] and J-STEPP [3] studies, pre-emptive therapy with oral doxycycline or minocycline, topical corticosteroid of weak potency and skin moisturizer were shown to exert favorable effects in preventing AfE with panitumumab therapy, although the effects of topical corticosteroid monotherapy on AfE remain unclear. Because of concerns regarding steroidinduced dermatitis (SID) [4] due to topical corticosteroid for facial lesions, weak- or medium-potency corticosteroids have been used for FAfE in clinical practice. However, the efficacy of topical corticosteroids of weak potency controlling FAfE activity is sometimes unsatisfactory. Recently, rapid 
effects have been reported from starting topical therapy with a very strong corticosteroid [5]. In addition, distinguishing between SID and FAfE due to EGFR inhibitors is not easy for oncologists treating CRC patients without consulting dermatologists.

The present study was designed to compare two regimens of topical corticosteroid therapy for AfE due to EGFR inhibitors among patients with CRC or NSCLC, with a focus on facial lesions (A phase III, open-label, randomized trial evaluating topical corticosteroid for Facial Acneiform dermatitis by EGFR Inhibitors: Stepwise rank down from potent corticosteroid: FAEISS study, NCCH1512). The regimens comprised one with serial ranking down from a very strong corticosteroid, and one with serial ranking up from a weak corticosteroid. Here, we present the primary analysis of metastatic CRC patients who received cetuximab or panitumumab. The results in NSCLC patients have been published in a separate paper [6].

\section{Materials And Methods}

\section{Patients}

Eligible patients were those with RAS wild-type metastatic CRC who received anti-EGFR antibody drugs (cetuximab or panitumumab). Other eligibility criteria included: age, 20-79 years; Eastern Cooperative Oncology Group Performance Status [7] score 0 or 1; absence of active bacterial, fungal and viral infections; and absence of any facial lesions influencing the evaluation of facial lesions. Informed consent for participation in this prospective study were obtained prior to enrolment. Patients with history of previous anti-EGFR therapy were excluded.

\section{Study design}

This study was an open-label, multicenter, randomized controlled trial. As the first registration for screening before randomization, eligible patients started pre-emptive therapy with oral minocycline at 100 $\mathrm{mg}$ or $200 \mathrm{mg}$ daily, and application of skin moisturizer containing heparinoid to the face twice daily from the date of starting anti-EGFR antibody therapy. All screened patients were instructed to avoid solar ultraviolet radiation, and use of sunscreen was recommended. Patients were instructed to apply topical $0.5 \%$ prednisolone cream when they developed FAfE. Patients who developed FAfE grade 1 or 2 within 8 weeks were randomly assigned to one of two groups: a ranking-up (RU) group, or a ranking-down (RD) group. The topical corticosteroids used in this study are listed in Supplementary table S1. The RU group started topical therapy with $0.5 \%$ prednisolone cream. When the grade of FAfE was exacerbated as judged by the attending physician, the topical corticosteroid was ranked up to one class higher. In contrast, the RD regimen started with a topical very strong corticosteroid, then corticosteroid was changed to one rank lower every 2 weeks. If the grade of FAfE exacerbated, the class of topical corticosteroid was maintained without ranking down (Fig. 1). In both groups, topical corticosteroid of strong or very strong class were used for AfE on non-face areas. If Grade 3 or more AfE affecting the whole body according to Common Terminology Criteria for Adverse Events (CTCAE) version 4.0-JCOG (Japan Clinical Oncology Group) was observed, protocol treatment was terminated. 


\section{Evaluations}

Efficacy variables were FAfE grade and patient QOL. For the assessment of endpoints, FAfE was graded after the end of the 8-week treatment by three trained dermatologists (central review) based on digital photographs of the face taken under a pre-determined, standardized method on each evaluation date. According to the qualitative scheme described by Scope et al[8] with the following minor modification, FAfE was classified as: grade 0, no symptoms; grade 1, less than one-third of the face involved; grade 2, more than one-third but less than two-thirds of the face involved; or grade 3, more than two-thirds of the face involved. QOL was assessed using the Dermatology Life Quality Index (DLQI) questionnaire immediately before and after completing 8 weeks of topical corticosteroid therapy.

Safety variables were specified as topical corticosteroid- and EGFR inhibitor-induced adverse events (AEs). AEs due to topical corticosteroid were also assessed by 3 dermatologists based on the digital photographs of the face according to the following criteria for SID and topical (bacterial/fungal/viral) infection. SID was assessed as: grade 0 , no symptoms; grade 1 , mild symptom, not interfering with daily life; grade 2, moderate symptom, partially interfering with daily life; or grade 3 , severe symptom, severely interfering with daily life. Topical infection was assessed as: grade 0 , no infection; grade 1 , involved lesion affects less than one-third of the area of face receiving topical treatment; grade 2, involved lesion affects more than one-third and less than two-thirds of the area of the face receiving topical treatment, and requires use of antibacterial medication; or grade 3: involved lesion affects more than two-thirds of the area of the face receiving topical treatment, and requires use of intravenous antimicrobial agents. The severity of EGFR inhibitor-induced AEs of the skin (i.e., pruritus, paronychia and xerosis) and AfE of whole body were assessed according to CTCAE version 4.0-JCOG by an attending physician.

Efficacy and safety variables were evaluated at the time of randomization (i.e., on the start date) and at 2 , 4, 6 and 8 weeks after starting randomized topical corticosteroid therapy, with the exception of the aforementioned QOL assessments.

\section{Study objectives (endpoints)}

On central review, presence or absence of FAfE grade 2 or higher at every evaluation date during the 8weeks study period was checked, and the total number of times FAfE was observed was evaluated as the primary endpoint. Secondary endpoints were the incidences of FAfE of grade 2 or higher, FAfE of grade 3 or higher and AEs due to topical corticosteroid during the study period. The numbers of patients who continued EGFR therapy, with FAfE of grade 1 or higher and with non-lowered QOL scores at the end of the study period were also evaluated.

\section{Statistical analyses}

A superiority test design was applied to determine whether the duration of grade 2 or greater FAfE during the study period was shorter in the RD group compared to the RU group. The total number of times FAfE grade 2 or higher was observed in the 8-week study (5 evaluation points) was compared between groups using the stratified Wilcoxon rank-sum test, with drug and sex as stratification factors. To test the null 
hypothesis (i.e., that there are no differences between groups in terms of the primary endpoint) using the stratified Wilcoxon rank-sum test with a one-sided alpha of 0.05 and $80 \%$ power, 94 patients were required for the primary analysis. Assuming the incident proportion of FAfE (grade 1 or higher) as $85 \%$, a sample size of 120 patients was planned for the first registration. Although this study was initially planned to include NSCLC patients, due to the low incidence of FAfE from EGFR-TKI treatment, registration of NSCLC patients was closed early and the result of that subgroup were published independently [6]. The incidences of FAfE of grade 2 or higher, and of FAfE grade 3 or higher, and the proportions of continued EGFR therapy, FAfE grade 1 or higher, and non-lowered QOL scores at the end of the study were evaluated using Fisher's exact test. Incidence of AEs due to topical CS during the 8-week study period were compared between RU and RD groups using Fisher's exact test. In addition, subgroup analyses for drug (cetuximab or panitumumab), additional anticancer drugs, sex and age (less than vs. greater than or equal to the median) were performed. One-sided $\mathrm{P}<0.05$ was considered statistically significant for analysis of the primary endpoint. Statistical analyses were performed using $S A S \circledast$ version 9.4 software (SAS Institute, Cary, NC).

\section{Results}

\section{Patients}

Between October 2016 and December 2018, a total of 172 patients with metastatic CRC were enrolled at the first registration and received pre-emptive therapy. All 106 patients who developed grade 1 or 2 facial AfE were randomized to the RU group $(n=53)$ or RD group $(n=53)$. Baseline characteristics were well balanced between groups (Table 1 ) and the proportion of grade 2 or higher FAfE was also similar between the RU group (11.3\%) and RD group (9.8\%). Two patients in the RD group terminated EGFR inhibitor therapy due to progression of the primary disease (untreated patients in Fig. 2). In the RD group, 1 patient judged as grade 3 by central review was ineligible. In the RU group, the potency of corticosteroid was ranked up to medium class in 2 patients before randomization within 2 weeks after initiating EGFR inhibitor therapy because of exacerbated FAfE.

In the RD group, 16 patients failed stepwise rank down due to exacerbation of FAfE at the 8-week evaluation (Table 2). The strength of corticosteroid was ranked up to medium in 10 patients and strong in 2 patients at the 8-week evaluation in the RU group (Table 2).

\section{Efficacy}

As the primary endpoint, no significant difference in the total number of observation of grade 2 or higher FAfE was seen between groups, using anticancer agents and sex as stratification factors $(p=0.86)$ (Table 3). The incidence of FAfE grade 2 or higher increased in a time-dependent manner. In the RU group, $11.3 \%, 26.1 \%, 32.6 \%, 35.0 \%$, and $35.7 \%$ of patients had developed FAfE grade 2 or higher at weeks 0 (second registration), 2, 4, 6, and 8 of the study, respectively. In the RD group, 9.8\%, 15.7\%, 34,1\%, 39\%, and $53.8 \%$ developed FAfE grade 2 or higher at the corresponding weeks (Table 4). Incidences of grade 2 or higher FAfE and grade 3 or higher FAfE during 8 weeks, continuation of EGFR therapy and grade 1 or 
higher FAfE at the end of protocol treatment showed no differences between groups (Supplementary table S2).

\section{QOL}

Few patients showed no worsening in QOL scores either group, and no significant difference was observed in the proportion without worsening of QOL scores between groups (Supplementary table S2).

\section{Safety}

Before randomization, 2 patients discontinued the pre-emptive therapy because of pruritus or liver dysfunction, probably caused by minocycline. After randomization, 2 patients in the RU group terminated protocol treatment due to grade 3 diarrhea and grade 3 anorexia caused by oral minocycline. One patient in the RD group terminated treatment due to local infection of the face, which was suspected to be due to topical corticosteroid. However, no difference in incidences of AEs caused by topical corticosteroid (i.e., SID and local infection) were seen between groups (Supplementary table S3).

\section{Discussion}

In the present study, pre-emptive therapy with oral minocycline and skin moisturizer on the face were started simultaneously with EGFR inhibitors, and topical $0.5 \%$ prednisolone cream, as a weak corticosteroid, was permitted for use against FAfE that developed before randomization. Grade 1 or grade 2 FAfE developed in 106 of 172 subjects (61.6\%) before randomization. This incidence is lower than those of previous reports without pre-emptive therapy, in which $90 \%$ with panitumumab and $80-86 \%$ with cetuximab developed AfE at some site on the body [9]. Favorable effects from the pre-emptive therapy on FAfE were suggested.

The duration of grade 2 or higher FAfE during the 8-week study period was assessed as the number of observations among the 5 observation timepoints for primary endpoint. The results showed no significant difference between RU and RD groups, and we could not identify any advantage of starting with a very strong corticosteroid. Moreover, no significant differences were seen between regimens in secondary endpoints, as the incidence of grade 2 or higher or grade 3 or higher FAfE during the study period, proportions with continuation of EGFR inhibitors, grade 1 or higher FAfE, or the percentage of patients with no worsening of QOL at the end of the study.

The RD regimen showed more favorable effects during the first 2 weeks but could not maintain control later and more than $50 \%$ of patients showed grade 2 or higher FAfE by the end of study. The severity of FAfE could potentially have increased over time in some patients, probably because the initial pre-emptive treatment with oral minocycline, and FAfE failed to maintain control in the RD group later in the treatment period, because the strength of topical corticosteroid was weakened in accordance with the protocol. This also suggested that topical corticosteroids offering strong potency or above are effective in controlling 
FAfE, whereas topical corticosteroids of medium potency or below might be inadequate to control FAfE in some severe cases.

Long-term use of stronger topical corticosteroids on the face carries a risk of AEs including SID and secondary infection, so we used a stepwise-ranking down every 2 weeks from a very strong corticosteroid to a weak corticosteroid unless exacerbation developed. Grade 1 SID was observed in 1 patient in the RU regimen. Grade 1 SID in 2 patients, and grade 2 local infection in 1 patient were observed in the RD regimen, but no marked differences were seen between regimens.

These results were based on an evaluation that lasted only 8 weeks. However, some patients resistant to strong topical corticosteroid for FAfE were present, particularly in the RD group, and these cases require careful attention to avoid SID. With the RU regimen, FAfE increased over time, but was limited to around $35 \%$ with some increase in corticosteroid potency by the end of the study.

\section{Conclusion}

Pre-emptive therapy with oral minocycline and skin moisturizer showed some promise for reducing the risk of grade 2 FAfE. Stepwise down-ranking from a very strong topical corticosteroid for FAfE worked during the first 2 weeks, but failed to show significant advantages over the RU regimen from a weak corticosteroid through the 8 weeks of the study. Short-term use of a very strong corticosteroid may have some merit for suppressing initial FAfE, but a standard RU regimen from a weak corticosteroid appears suitable in long-term care for FAfE and should be considered the standard treatment for FAfE due to EGFR inhibitor therapy.

\section{Limitations}

The duration of protocol treatment was only 8 weeks and no follow-up period was provided afterword. Long-term observation of the duration and severity of FAfE, the strength of the topical corticosteroid needed, and the AEs of topical corticosteroids on the face is necessary.

\section{Declarations}

\section{Acknowledgement}

The authors are grateful to their patients and the staff of all participating institutions. The authors also wish to thank Ms. Kaoru Koike, Mr. Tomoaki Yamada,and Ms. Keiko Ohata for assistance with data management, and Ms. Harumi Kaba for designing the case report forms and electronic data-capture system.

\section{Funding}


This study was supported by Japan Agency for Medical Research and Development (AMED) under Grant Number JP17ck0106326.

\section{Conflicts of interests}

All authorshave no conflicts of interests to reports for this paper.

\section{Ethics approval}

This study was conducted according to the Declaration of Helsinki and was approved by the ethics review board of each participating institution. Research ethics boards at all participating institutions approved the study protocol.

\section{Consent to participate}

Each enrolled patient provided written informed consent.

\section{Consent for publication}

Not applicable

\section{Availability of data and material}

All data generated or analyzed during this study are included in this published article.

\section{Code availability}

Not applicable

\section{Authors' contributions}

The study conceptualization and design:Katsuko Kikuchi, Yoshio Kiyohara, Naoya Yamazaki, Tetsuya Hamaguchi, Keiko Nozawa, Haruhiko Fukuda and Hitoshi Mizutani. Formal analysis and investigation: Taro Shibata, Ryunosuke Machida and Haruhiko Fukuda. Funding acquisition: Keiko Nozawa. Material preparation and data collection:Katsuko Kikuchi,Naoya Yamazaki, Keiko Nozawa, Tetsuya Hamaguchi,Atsuo Takashima,Hirokazu Shoji,NarikazuBoku,SumikoTakatsuka, TatsuyaTakenouchi, TomohiroNishina, Shusuke Yoshikawa, Masanobu Takahashi, AkikoHasegawa, Akihito Kawazoe,ToshikiMasuishi and Yoshio Kiyohara.The first draft of the manuscript was written by Katsuko Kikuchi. All authors contributed comments and helped revise previous versions of the manuscript. Allauthors read and approved the final manuscript.

\section{References}

1. Agero AL, Dusza SW, Benvenuto-Andrade $C$ et al. Dermatologic side effects associated with the epidermal growth factor receptor inhibitors. J Am Acad Dermatol 2006; 55: 657-670. 
2. Lacouture ME, Mitchell EP, Piperdi B et al. Skin toxicity evaluation protocol with panitumumab (STEPP), a phase II, open-label, randomized trial evaluating the impact of a pre-Emptive Skin treatment regimen on skin toxicities and quality of life in patients with metastatic colorectal cancer. $\mathrm{J}$ Clin Oncol 2010; 28: 1351-1357.

3. Kobayashi Y, Komatsu Y, Yuki S et al. Randomized controlled trial on the skin toxicity of panitumumab in Japanese patients with metastatic colorectal cancer: HGCSG1001 study; J-STEPP. Future Oncol 2015; 11: 617-627.

4. Ljubojeviae S, Basta-Juzbasiae A, Lipozeneiae J. Steroid dermatitis resembling rosacea: aetiopathogenesis and treatment. J Eur Acad Dermatol Venereol 2002; 16: 121-126.

5. Yamazaki N, Kiyohara $\mathrm{Y}$, Kudoh $\mathrm{S}$ et al. Optimal strength and timing of steroids in the management of erlotinib-related skin toxicities in a post-marketing surveillance study (POLARSTAR) of 9909 nonsmall-cell lung cancer patients. Int J Clin Oncol 2016; 21: 248-253.

6. Nishino K, Fujiwara Y, Ohe Y et al. Results of the non-small cell lung cancer part of a phase III, openlabel, randomized trial evaluating topical corticosteroid therapy for facial acneiform dermatitis induced by EGFR inhibitors: stepwise rank down from potent corticosteroid (FAEISS study, NCCH1512). Support Care Cancer 2020.

7. Oken MM, Creech RH, Tormey DC et al. Toxicity and response criteria of the Eastern Cooperative Oncology Group. Am J Clin Oncol 1982; 5: 649-655.

8. Scope A, Lieb JA, Dusza SW et al. A prospective randomized trial of topical pimecrolimus for cetuximab-associated acnelike eruption. J Am Acad Dermatol 2009; 61: 614-620.

9. Overman MJ, Hoff PM. EGFR-targeted therapies in colorectal cancer. Dis Colon Rectum 2007; 50: 1259-1270.

\section{Tables}

Due to technical limitations, tables are only available as a download in the Supplemental Files section.

\section{Figures}




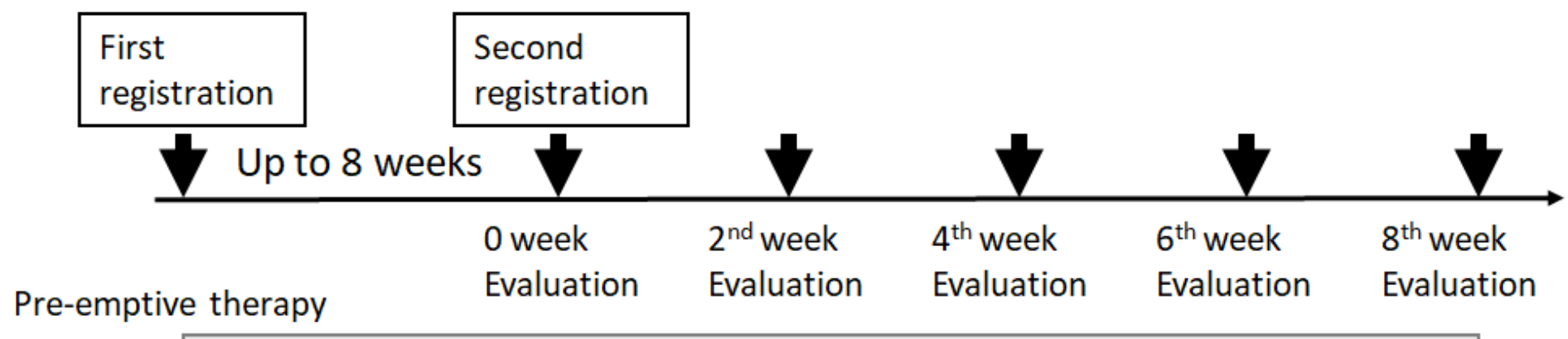

Oral minocycline 100 or $200 \mathrm{mg}$ daily and skin moisturizer

Ranking up*

regimen

\begin{tabular}{|l|l|l|l|}
\hline Weak & Weak & Weak & Weak \\
\hline
\end{tabular}

Ranking down** regimen

Very
strong

Medium

Weak

* If facial AfE grade was increased, the strength of topical corticosteroid was raised one rank.

** If facial AfE grade was increased, the same class of topical corticosteroid was continued.

Figure 1

Study schema. 


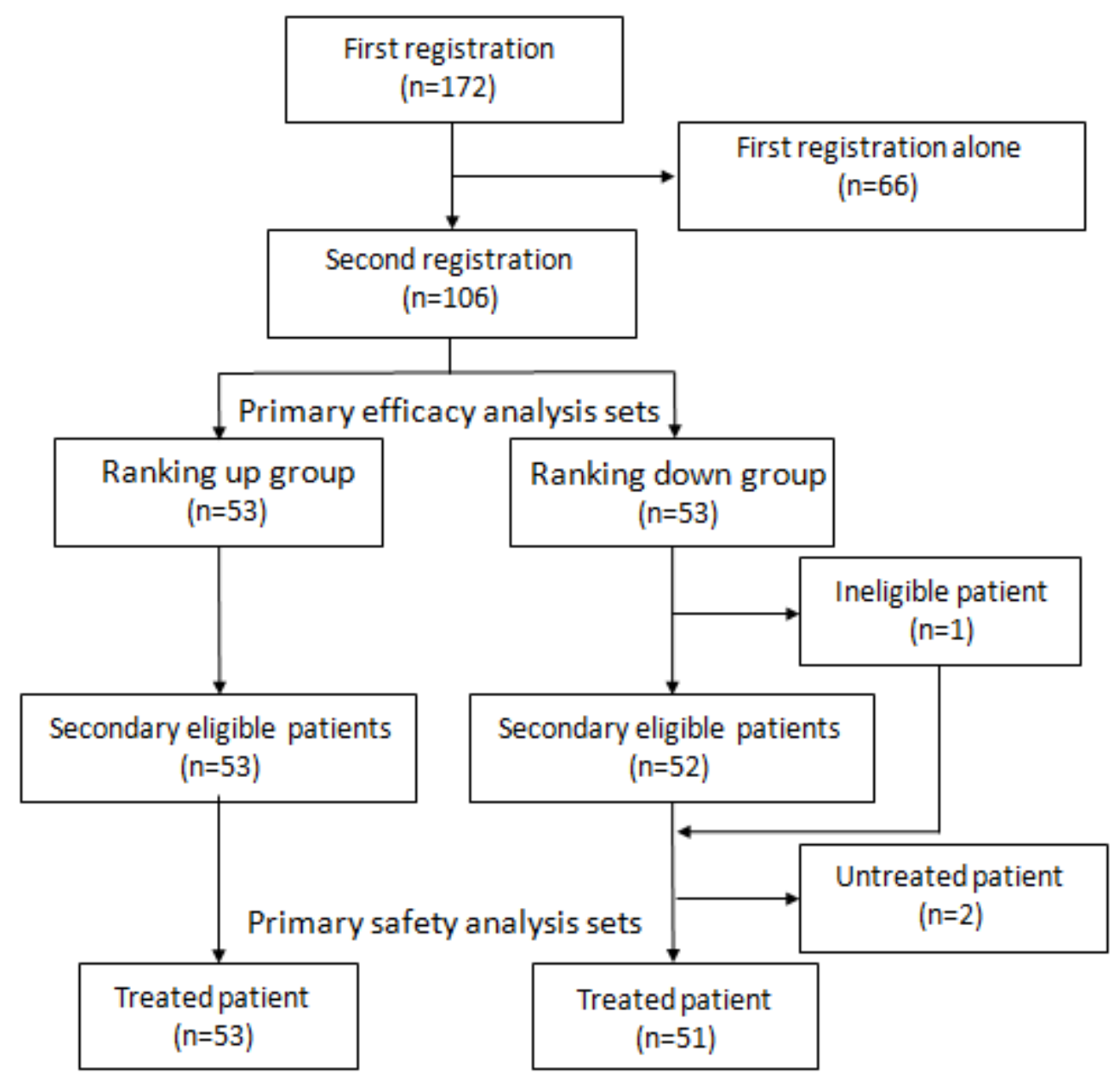

Figure 2

CONSORT diagram.

\section{Supplementary Files}

This is a list of supplementary files associated with this preprint. Click to download.

- FAEISSSuppTable0809.xlsx

- FAEISSTable0809.xIsx 